\title{
Design of photonic crystal horn antenna for transverse electric modes
}

\author{
Ehsan BEIRANVAND*, Mohammad DANAIE, MaJid AFSAHI \\ Faculty of electrical and computer engineering, Semnan University, Semnan, Iran \\ ${ }^{*}$ Corresponding author: beiranvand@semnan.ac.ir
}

\begin{abstract}
In this paper, by modifying defects in a photonic crystal lattice, a two-dimensional photonic crystal horn antenna is designed. The photonic crystal used for this purpose is composed of a hexagonal lattice of circular holes in a dielectric slab. The results of this paper allow us to design a photonic crystal antenna capable of separating TE and TM modes. The designed structure has a very simple design that allows low cost fabrication. The structure is numerically simulated using a finite-difference time-domain (FDTD) method. Its wide bandwidth, its low loss and the ability to transmit waves at a terahertz frequency range are the antenna's main advantages. The return loss for the frequency range of 180 to $215 \mathrm{THz}$ is from -6.63 to $-28.3 \mathrm{~dB}$. Moreover, a $35 \mathrm{THz}$ bandwidth is obtained for this structure.
\end{abstract}

Keywords: photonic crystal-based horn antenna, transverse electrical modes, FDTD method.

\section{Introduction}

Photonic crystal-based structures are widely used for design of radiative structures. The dielectric constant of photonic crystals is periodic in one, or two, or three dimensions. One of important features of these structures is the banned electromagnetic band for a frequency range which is referred to as the optical bandgap [1-4]

Nanotechnology opens the door towards a new communication paradigm that introduces a variety of novel tools. It enables engineers to design and manufacture nanoscale electronic devices and systems with substantially novel features [ [ $]$ ]. These devices can cover radio frequencies from the terahertz range and beyond, up to optical frequencies. The integration of nano-devices can lead to the design of nano-networks with a plethora of potential applications in the biomedical, industrial, environmental and military fields. Nevertheless, nano-networks are not just considered a mere downscaled version of conventional networks. The classical communication paradigms need to undergo subtle revisions before being applied to such scenarios []ㅡ. Up to date, enabling the communication among nano-devices is still a challenging issue. The miniaturization of classical antennas in order to meet size requirements of nano-devices requires using very high frequencies, which results in significant propagation loss. Moreover, the in- 
trinsic behavior of metals can be different at the nanoscale dimensions. Thus, common assumptions in the antenna theory such as the ideal perfect electric conductor (PEC), may not hold in nanoscale dimensions [7].

Photonic crystals are one of the platforms that can be used for designing optical components. These structures allow for the confinement of optical pulses and control of light emission in very small and compact spaces [ [ $]$ ]. In recent years, various photonic crystal components have been designed which can be used in optical communication. These include optical filters $[\underline{9}, \underline{10}]$, optical multiplexers [11], optical switches $[\underline{12}, \underline{13}]$, optical interconnects [14], imaging devices [15], optical sensors [16] and optical gates [17-19]. The combination of photonic crystal structures with plasmonic or graphene devices creates a variety of new possibilities [ $\underline{20}-\underline{22}]$.

Photonic crystal structures have long been used for the transmission of electromagnetic waves and they are widely used in optical telecommunications. These periodic structures are usually simulated using a finite-difference time-domain (FDTD) method [23] There are many articles on this topic, which have compared the results of experiments with simulations. The accuracy of the simulation results in these articles assures us that the FDTD results are close to experimental results [24-28]. As mentioned above, the two-dimensional slab photonic crystal structures can be analyzed and simulated by numerical methods such as FDTD. In this paper, the RSOFT software is also used for analysis and FDTD simulations. As previous studies show, it can be said that the design and simulation results in this paper will be in agreement with the experimental results.

This study has shown the possibility of design and fabrication of 2-D photonic crystals antennas with specified frequency-dependent transmission. The results of this paper allow us to design a photonic crystal antenna capable of separating TE and TM modes. The separation of TE and TM modes can be achieved by appropriately designing the photonic crystal waveguides and the photonic crystal lattice structure. The horn structure reduces the return loss of the waves.

There are some methods proposed in the literature to increase the bandwidth of photonic crystal waveguides and splitters [29-31]. The first use of photonic crystal-based structures in antennas was for increasing the antenna's radiation efficiency [32], which triggered the widespread use of photonic crystal-based structures for antennas and telecommunications devices. By introducing defects in the photonic crystalline structure, a resonator can be created, which is used in crystal-based antennas in either one [33], two [34] or three [35] dimensions. They can also be used to remove the interactional and destructive couplings between the antennas [하] $]$. As a result, antennas with high efficiency and directionality can be obtained. In recent years, EBGs and PBGs have been highly used in order to improve the radiation characteristics of antennas [ $\underline{37}-\underline{42}$ ].

The first photonic crystal horn antennas were presented in $[43,44]$. The idea was later expanded, as functional structures were designed in three dimensions [는 $\underline{50}]$. Considering these and the preceding issues mentioned above, photonic crystal-based antennas have been considered by researchers in recent years. Furthermore, various antennas are designed with different applications for different uses. The integration of such antennas with other components paves the way for future all-optical systems [1ㅡ-53] 
Horn antennas for transverse electric (TE) waves have not been designed yet. Here, the antenna is designed for TE modes using a dielectric slab in which circular holes are etched. Furthermore, parametric studies on changes in antenna length and antenna's aperture angle, as well as the type of arrangement of the elements are performed. By determining the photonic crystal structure, the possibility of propagating waves with minimal loss is investigated.

\section{Photonic crystal-based horn antenna design}

\subsection{Waveguide array design}

First, the photonic crystal structure is designed using a square array of holes with a radius of $0.4 a$. Here $a$ is the lattice constant. In this structure, we need to etch holes in a dielectric slab with a dielectric coefficient of 11.56 according to Fig. 1. The first step to design a suitable waveguide is to compute the bandgap of the photonic crystal. The Brillouin zone of the optical bandgap of the photonic crystal is indicated in Fig. 2. The waveguide modes associated with Fig. 1 are depicted in Fig. 3a. As shown in Fig. 3b, a single mode exists for the normalized frequency range of 0.25 to $0.29(a / \lambda)$.

We next examined the optical group index to investigate the light emission in the photonic crystal waveguide structure. Figure $3 \mathbf{b}$ shows a part of the propagation mode

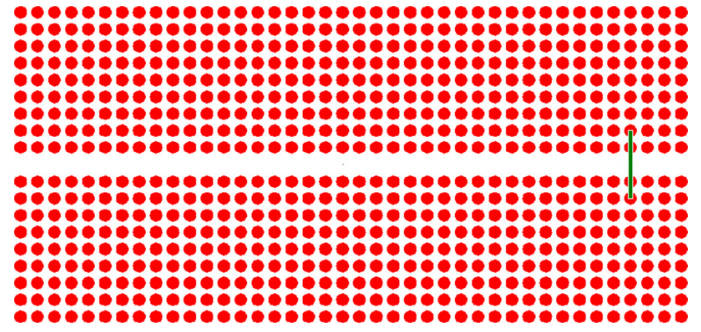

Fig. 1. The photonic crystal waveguide designed by removing a row of holes.

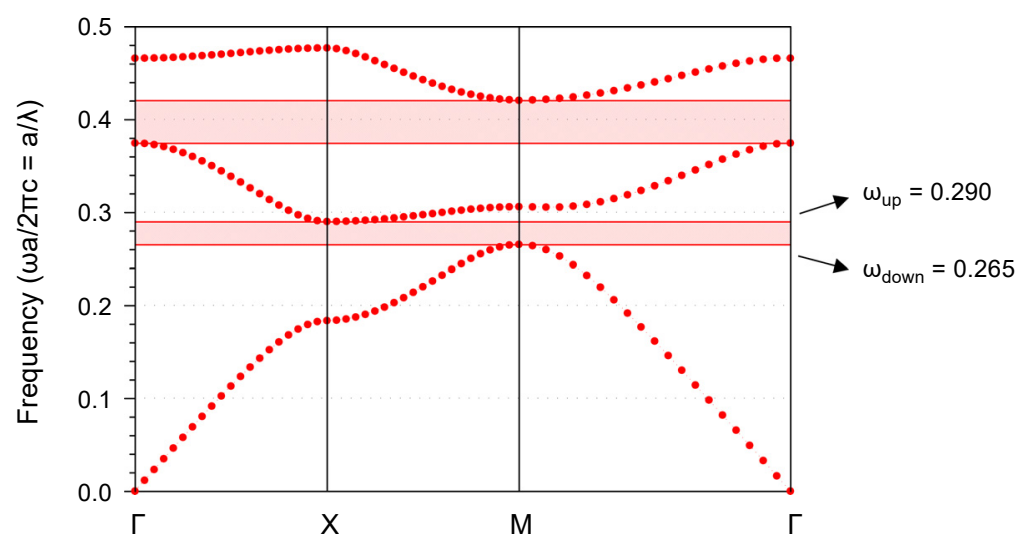

Fig. 2. The Brillouin zone for the photonic crystal structure depicted in Fig. 1. 

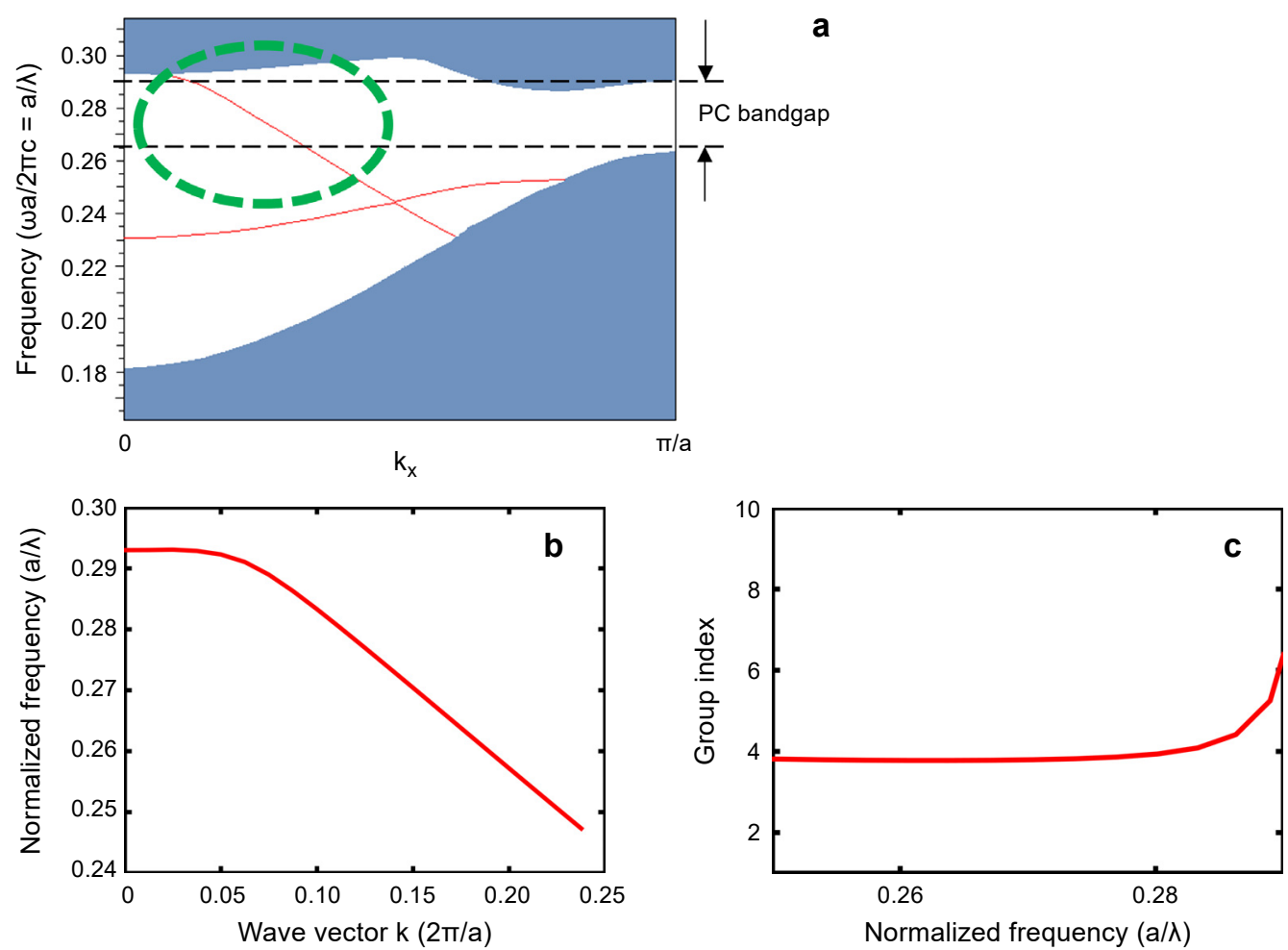

Fig. 3. Transitional electrical mode inside the photonic crystal-based waveguide. Mode propagated within the waveguide (a). Part of the propagation mode that is placed within the band gap (b). Optical group index of waveguide structure (c).

of Fig. 3a, which is marked with the dashed line. Figure 3c shows the optical group index. This figure shows that the speed of light is four times higher.

\subsection{Designing the photonic crystal-based horn antenna}

In the following, to design the photonic crystal horn antenna, the structure of the photonic crystal waveguide, as shown in Fig. 4, is designed with periodic photonic crystal struc-

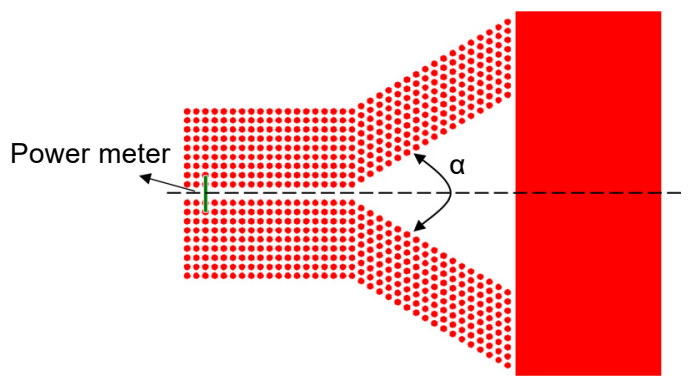

Fig. 4. Photonic crystal-based horn antenna. 
tures, with a two-dimensional dielectric slab with a dielectric coefficient of 11.56. The air holes in dielectric slab are marked in red. As mentioned earlier, at the output of the horn antenna, is placed on an air slab. The wave propagates in the air after passing through the antenna. It is necessary to calculate the photonic bandgap of the photonic crystal structure. Then we calculated the TE modes, which expand in photonic crystal waveguide.

With this method, we design a suitable waveguide for wave propagation, and we can use a photonic crystal horn antenna by applying changes to the layout of arrays, which gives us good scattering parameters. Figure 5 shows the antenna return loss because for antenna aperture angle $\alpha$ changes which is from 0 to $50^{\circ}$. Figure 6 shows a return losses average for different $\alpha$ values.

Figure 7 shows the return loss for $\alpha=50^{\circ}$, which has the best result for the photonic crystal horn antenna. In Fig. 8, it has been shown that the return losses from 180 to

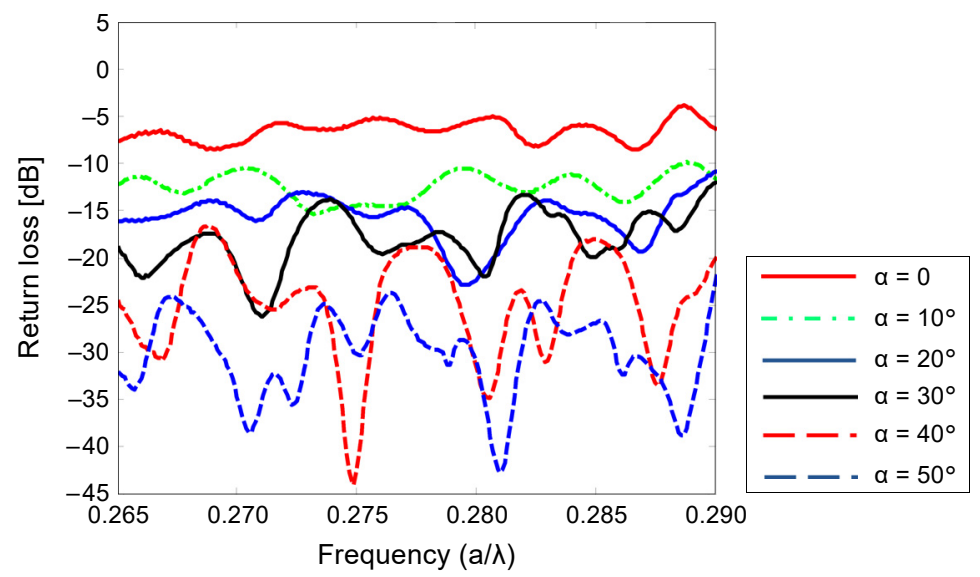

Fig. 5. Return loss of the horn antenna for different values of antenna aperture angle $\alpha$ for the antenna as shown in Fig. 4.

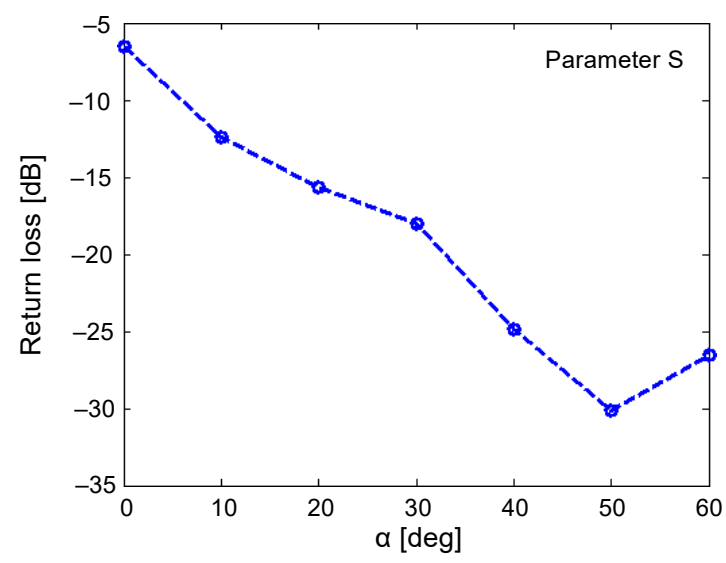

Fig. 6. Return loss average for different values of antenna aperture angle $\alpha$ for the antenna as shown in Fig. 4. 


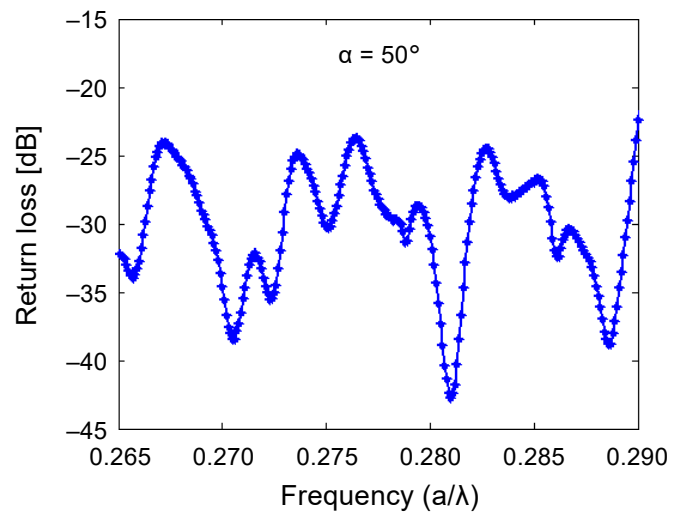

Fig. 7. Antenna return loss for $\alpha=50^{\circ}$.

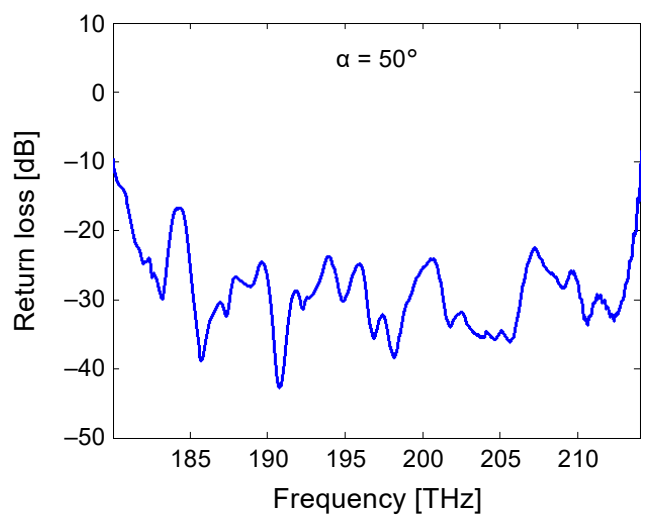

Fig. 8. Antenna return loss for $\alpha=50^{\circ}$.

$215 \mathrm{THz}$ are less than $-10 \mathrm{~dB}$ which provided the bandwidth $35 \mathrm{THz}$ with an average return loss of $-28.3 \mathrm{~dB}$.

After investigating the angle of the antenna, the length of the antenna was examined. By changing the length of the antenna, the results of the return loss and other antenna transmission parameters were changed. Figure 9 shows the photonic crystal

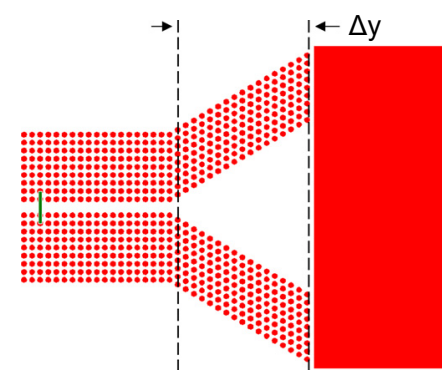

Fig. 9. Photonic crystal horn antenna with antenna length variations. 


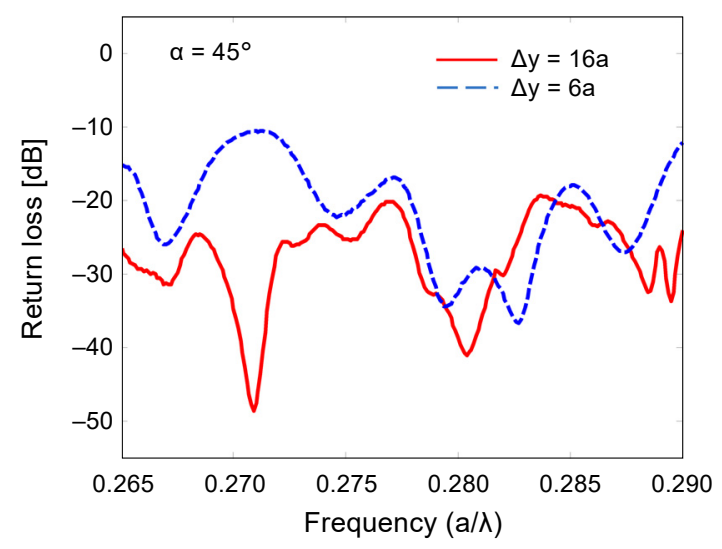

Fig. 10. Antenna return loss in $\Delta y=16 a$ and $\Delta y=6 a$ for the antenna as shown in Fig. 9.

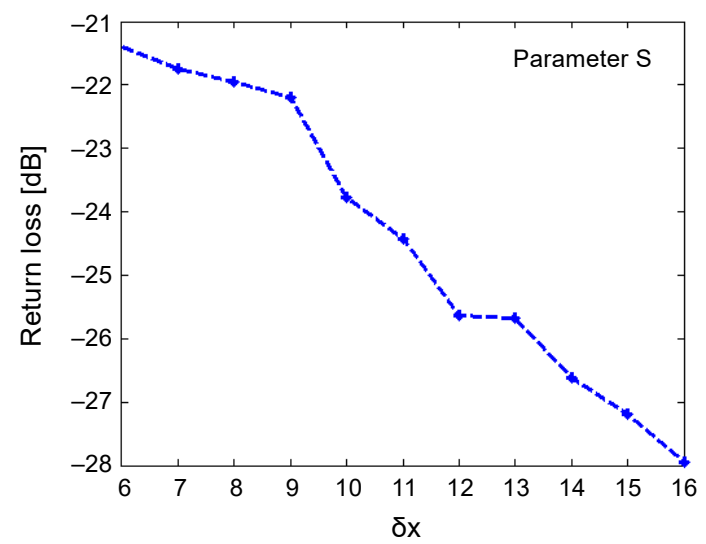

Fig. 11. Average return losses of the antenna in different values of $\Delta y$ for the antenna as shown in Fig. 9.

horn antenna that has been investigated by the parameter $\Delta y$ (antenna outlet length). By study this parameter, we reach a linear relationship between the antenna length and the return loss. As the antenna length increases, the return loss decreases. As the length of the structure of the photonic crystal horn antenna increases, the path loss and return losses increase. The antenna should be considered in an optimal length, so that the antenna power output is acceptable and the return losses will be reduced. The result of this review is below. Figure 10 shows the return losses of the photonic crystal horn antenna for $\Delta y=16 a$ and $\Delta y=6 a$. Figure 11 shows the average return losses for different values of $\Delta y$.

Given the tools available to the fabrication of photonic crystal structures, we investigated the accuracy of the fabrication and the impact of fabrication errors by adopting different values for the hole radius size in numerical simulation. Figures 12 and 13 show different values of return losses for different values of the hole radius. These figures clearly show that the amount of return losses will have acceptable tolerances. 


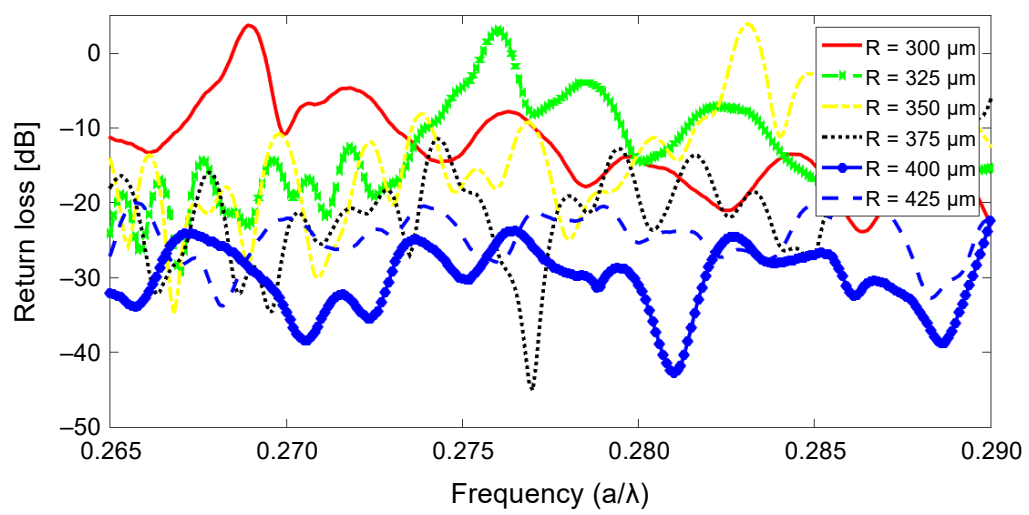

Fig. 12. Comparison of return losses for different values of holes radius in structure frequency band.

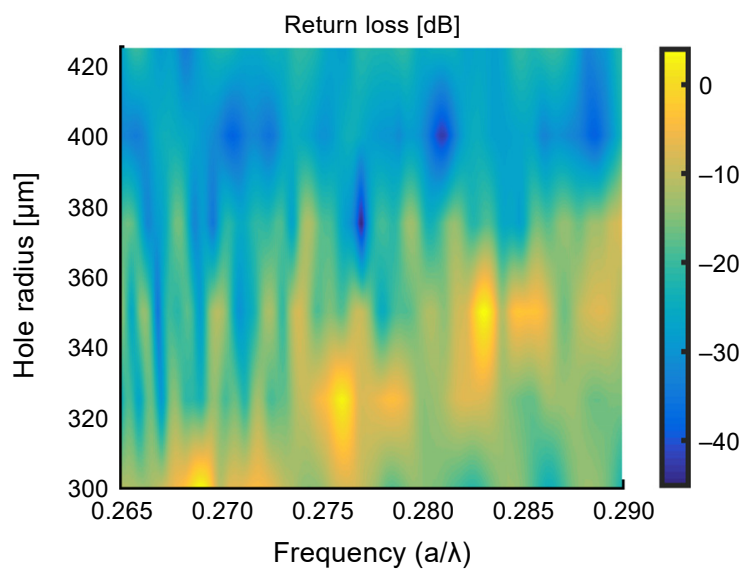

Fig. 13. Diagram of antenna return losses for different values of the holes radius $(300 \mu \mathrm{m}<R<425 \mu \mathrm{m})$.

According to the results of the return losses of the designed photonic crystal structure, the amount of losses will be very small. The amount of return losses in the optimized state is $-28 \mathrm{~dB}$.

We then developed the photonic crystal horn antenna for the hexagonal lattice, whose results are summarized below. The results obtained from this structure revealed

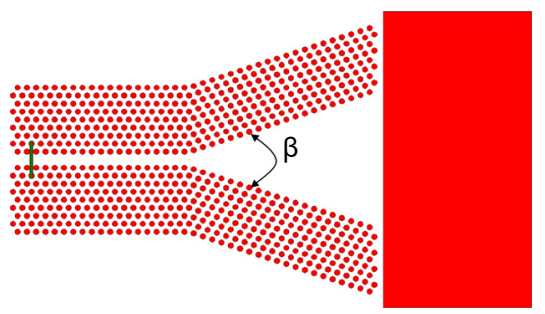

Fig. 14. Photonics crystal horn antenna with hexagonal lattice. 
that the square grid is more suitable for this structure. Although we were faced with a reduction in return losses, the return losses were higher compared to the square grid. The photonic crystal horn antenna with a hexagonal lattice is shown in Fig. 14. In addition, the radius of air holes shown in red is $0.4 \mathrm{~mm}$. The basic slab is made of die-

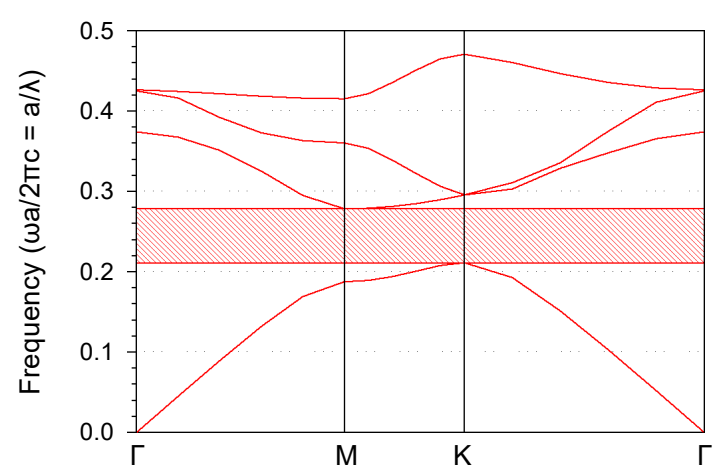

Fig. 15. Bandgap diagram of hexagonal structure shown in Fig. 14.

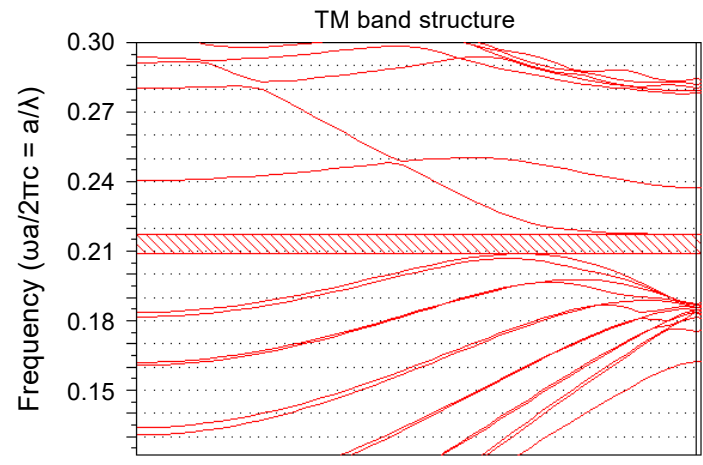

Fig. 16. Transitional transverse modes for hexagonal lattice structure shown in Fig. 14.

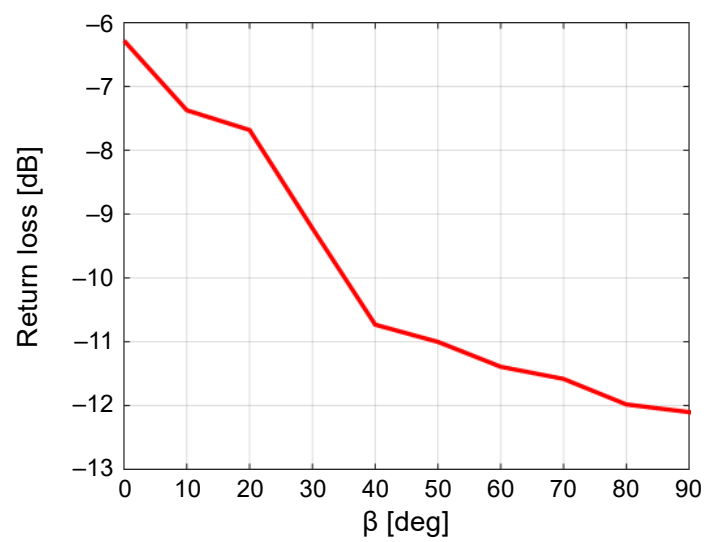

Fig. 17. Changes in $\beta$ angle in the hexagonal lattice structure shown in Fig. 14. 


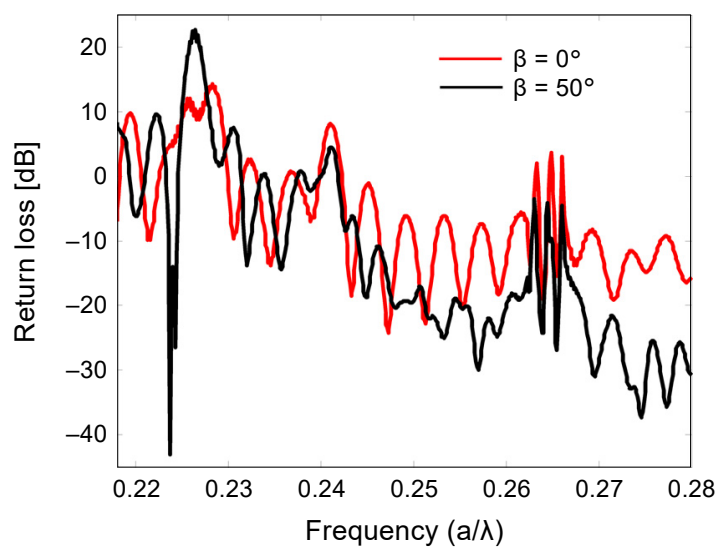

Fig. 18. Return losses for the photonic crystal horn antenna by hexagonal lattice for two values of $\beta$ at $0^{\circ}$ and $50^{\circ}$.

lectric with a dielectric coefficient of 11.56. In Fig. 15, the band gap diagram of the photonic crystal structure for the hexagonal lattice is indicated, and the transverse electric waveguide mode that extends within the waveguide is shown in Fig. 16. The results of the return losses relative to the angles of the antenna are shown in Fig. 17, and Fig. 18 shows the return losses of the antenna with the hexagonal lattice for the two values of $\beta$ at $0^{\circ}$ and $50^{\circ}$.

\section{Conclusions}

In this paper, the possibility of design and fabrication of 2-D photonic crystals antenna has been investigated. The results of this paper allow us to design a photonic crystal antenna capable of separating TE and TM modes. The separation of specific TE and TM modes is achieved by appropriately designing the photonic crystal waveguides and the photonic crystal lattice structure. The horn antenna structure increases the transmission power and reduces the return losses of the waves. Designed structure has a very simple design that allows low cost fabrication and low tolerance. It also transmits optical waves in a wide frequency range while still being simple. Photonic crystal horn antenna is designed for transverse electric waves for the first time. Using the photonic crystal horn antenna, the return losses average in a simple waveguide for the frequency range 180 to $215 \mathrm{THz}$ was -6.63 to $-28.3 \mathrm{~dB}$. Moreover, that $35 \mathrm{THz}$ bandwidth was obtained for this structure. In this paper, three parameters of the antenna such as antenna length, antenna aperture angle and the photonic crystal lattice of elements were investigated, and the results indicate that this structure is suitable for wave transfer.

\section{References}

[1] Meade R.D., Brommer K.D., Rappe A.M., Joannopoulos J.D., Photonic bound states in periodic dielectric materials, Physical Review B 44(24), 1991, p. 13772(R), DOI: 10.1103/PhysRevB.44.13772.

[2] Joannopoulos J.D., Meade R.D.,Winn J.N., Photonic Crystals, Princeton University Press, NJ, 1995. 
[3] Joannopoulos J.D., Villeneuve P.R., Fan S., Photonic crystals: putting a new twist on light, Nature 386, 1997, pp. 143-149, DOI: 10.1038/386143a0.

[4] Ho K.M., Chan C.T., Soukoulis C.M., Existence of a photonic gap in periodic dielectric structures, Physical Review Letters 65(25), 1990, p. 3152, DOI: 10.1103/PhysRevLett.65.3152.

[5] RUSSER P., FICHTNER N., Nanoelectronics in radio-frequency technology, IEEE Microwave Magazine 11(3), 2010, pp. 119-135, DOI: 10.1109/MMM.2010.936077.

[6] JoRNET J.M., AKYILDIZ I.F., Graphene-based plasmonic nano-antenna for terahertz band communication in nanonetworks, IEEE Journal on Selected Areas in Communications 31(12), 2013, pp. 685-694, DOI: 10.1109/JSAC.2013.SUP2.1213001.

[7] Burke P.J., Li S., Yu Z., Quantitative theory of nanowire and nanotube antenna performance, IEEE Transactions on Nanotechnology 5(4), 2006, pp. 314-334, DOI: 10.1109/TNANO.2006.877430.

[8] Joannopoulos J.D., Johnson S.G., Winn J.N., Meade R.D., Photonic Crystal Molding Flow of Light, Princeton University Press, Princeton, NJ, USA, 2008.

[9] Moradi M., Danaie M., Orouj A.A., Design and analysis of an optical full-adder based on nonlinear photonic crystal ring resonators, Optik 172, 2018, pp. 127-136, DOI: 10.1016/j.ijleo.2018.07.016.

[10] Danaie M., Geravand A., Mohammadi S., Photonic crystal double-coupled cavity waveguides and their application in design of slow-light delay lines, Photonics and Nanostructures - Fundamentals and Applications 28, 2018, pp. 61-69, DOI: 10.1016/j.photonics.2017.11.009.

[11] Khani S., Danaie M., Rezaei P., Double and triple-wavelength plasmonic demultiplexers based on improved circular nanodisk resonators, Optical Engineering 57(10), 2018, article 107102, DOI: 10.1117/1.OE.57.10.107102.

[12] Sharkawy A., Shi S., Prather D.W., Soref R.A., Electro-optical switching using coupled photonic crystal waveguides, Optics Express 10(20), 2002, pp. 1048-1059, DOI: 10.1364/OE.10.001048.

[13] Danaie M., Kaatuzian H., Design of a photonic crystal differential phase comparator for a Mach -Zehnder switch, Journal of Optics 13(1), 2010, article 015504, DOI: 10.1088/2040-8978/13/1/ 015504.

[14] Zhang X., Hosseini A., Lin X., Subbaraman H., Chen R.T., Polymer-based hybrid-integrated photonic devices for silicon on-chip modulation and board-level optical interconnects, IEEE Journal of Selected Topics in Quantum Electronics 19(6), 2013, pp. 196-210, article 3401115, DOI: 10.1109 / JSTQE.2013.2268386.

[15] Yu J., Zeng H., Lui H., Skibina J.S., Steinmeyer G., Tang S., Characterization and application of chirped photonic crystal fiber in multiphoton imaging, Optics Express 22(9), 2014, pp. 10366-10379, DOI: $10.1364 /$ OE.22.010366.

[16] Zhang X., Hosseini A., Subbaraman H., Wang S., Zhan Q., Luo J., Jen A.K.Y., Chen R.T., Integrated photonic electromagnetic field sensor based on broadband bowtie antenna coupled silicon organic hybrid modulator, Journal of Lightwave Technology 32(20), 2014, pp. 3774-3784, DOI: 10.1109 / JLT.2014.2319152.

[17] Geravand A., Danaie M., Mohammadi S., All-optical photonic crystal memory cells based on cavities with a dual-argument hysteresis feature, Optics Communications 430, 2019, pp. 323-335, DOI: 10.1016/j.optcom.2018.08.052.

[18] Danaei M., KaAtuzian H., Design and simulation of an all-optical photonic crystal AND gate using nonlinear Kerr effect, Optical and Quantum Electronics 44(1-2), 2012, pp. 27-34, DOI: $10.1007 /$ s11082-011-9527-y.

[19] Zhang X., Wang Y., Sun J., Liu D., Huang D., All-optical AND gate at 10 Gbit/s based on cascaded single-port-coupled SOAs, Optics Express 12(3), 2004, pp. 361-366, DOI: 10.1364/OPEX.12.000361.

[20] Fan Z., Li S., Liu Q., An G., Chen H., Li J., Chao D., Li H., Zi J., Tian W., High sensitivity of refractive index sensor based on analyte-filled photonic crystal fiber with surface plasmon resonance, IEEE Photonics Journal 7(3), 2015, article 4800809, DOI: 10.1109/JPHOT.2015.2432079.

[21] Rifat A.A., Mahdiraj G.A., Sua1 Y.M., Shee Y.G., Ahmed R., Chow D.M., Adikan F.R.M., Surface plasmon resonance photonic crystal fiber biosensor: a practical sensing approach, IEEE Photonics Technology Letters 27(15), 2015, pp. 1628-1631, DOI: 10.1109/LPT.2015.2432812. 
[22] Rifat A.A., Ahmed R., Mahdiraji G.A., Adikan F.R.M., Highly sensitive D-shaped photonic crystal fiber-based plasmonic biosensor in visible to near-IR, IEEE Sensors Journal 17(9), 2017, pp. 2776 -2783, DOI: $10.1109 /$ JSEN.2017.2677473.

[23] Si L.M., Liu Y., Lu H.D., Sun H.J., Lv X., Zнu W., Experimental realization of high transmittance $\mathrm{THz} 90^{\circ}$-bend waveguide using EMXT structure, IEEE Photonics Technology Letters 25(5), 2013, pp. 519-522, DOI: 10.1109/LPT.2013.2244878.

[24] Zhao Y., GrischKowsKy D.R., 2-D terahertz metallic photonic crystals in parallel-plate waveguides, IEEE Transactions on Microwave Theory and Techniques 55(4), 2007, pp. 656-663, DOI: $\underline{10.1109 /}$ TMTT.2007.892798.

[25] Withayachumnankul W., Yamada R., Fujita M., Nagatsuma T., All-dielectric rod antenna array for terahertz communications, APL Photonics 3(5), 2018, article 051707, DOI: 10.1063/1.5023787.

[26] Withayachumnankul W., Fujita M., Nagatsuma T., Integrated silicon photonic crystals toward terahertz communications, Advanced Optical Materials 6(16), 2018, article 1800401, DOI: $10.1002 /$ adom.201800401.

[27] Furukado Y., Abe H., Hinakura Y., Baba T., Experimental simulation of ranging action using Si photonic crystal modulator and optical antenna, Optics Express 26(14), 2018, pp. 18222-18229, DOI: $10.1364 / \mathrm{OE} .26 .018222$.

[28] Frei W.R., Tortorelli D.A., Johnson H.T., Topology optimization of a photonic crystal waveguide termination to maximize directional emission, Applied Physics Letters 86(11), 2005, article 111114, DOI: $10.1063 / 1.1885170$.

[29] Danaie M., KaAtuZian H., Improvement of power coupling in a nonlinear photonic crystal directional coupler switch, Photonics and Nanostructures - Fundamentals and Applications 9(1), 2011, pp. 70-81, DOI: 10.1016/j.photonics.2010.10.002.

[30] Danaie M., Attari A.R., Mirsalehi M.M., Naseh S., Optimization of Two Dimensional Photonic Crystal Waveguides for TM polarization, [In] EUROCON 2007 - The International Conference on "Computer as a Tool", Warsaw, 2007, pp. 1218-1222, DOI: 10.1109/EURCON.2007.4400488.

[31] Danaie M., Nasiri-Far R., Dideban A., Design of a high-bandwidth Y-shaped photonic crystal power splitter for TE modes, International Journal of Optics and Photonics 12(1), 2018, pp. 33-42, DOI: $10.29252 /$ ijop.12.1.33.

[32] Brown E.R., Parker C.D., Y ablonovitch E., Radiation properties of a planar antenna on a photonic -crystal substrate, Journal of the Optical Society of America B 10(2), 1993, pp. 404-407, DOI: 10.1364/JOSAB.10.000404.

[33] Serier C., Cheype C., Chantalat R., Thevenot M., Monediere T., Reinex A., Jecko B., 1-D photonic bandgap resonator antenna, Microwave and Optical Technology Letters 29(5), 2001, pp. 312-315, DOI: $10.1002 /$ mop. 1164 .

[34] Cheype C., Serier C., Thevenot M., Monediere T., Reineix A., Jecko B., An electromagnetic bandgap resonator antenna, IEEE Transactions on Antennas and Propagation 50(9), 2002, pp. 1285-1290, DOI: $\underline{10.1109 / \text { TAP.2002.800699. }}$.

[35] Biswas R., Ozbay E., Temelkuran B., Bayindir M., Sigalas M.M., Ho K.M., Exceptionally directional sources with photonic-bandgap crystals, Journal of the Optical Society of America B 18(11), 2001, pp. 1684-1689, DOI: 10.1364/JOSAB.18.001684.

[36] Rajo-Iglesias E., Quevedo-Teruel Ó., Inclan-Sanchez L., Mutual coupling reduction in patch antenna arrays by using a planar ebg structure and a multilayer dielectric substrate, IEEE Transactions on Antennas and Propagation 56(6), 2008, pp. 1648-1655, DOI: 10.1109/TAP.2008.923306.

[37] Brown E.R., Parker C.D., Y ablonovitch E., Radiation properties of a planar antenna on a photonic -crystal substrate, Journal of the Optical Society of America B 10(2), 1993, pp. 404-407, DOI: 10.1364/JOSAB.10.000404.

[38] Gonzalo R., Ederra I., Mann C.M., De MaAgt P., Radiation properties of terahertz dipole antenna mounted on photonic crystal, Electronics Letters 37(10), 2001, pp. 613-614, DOI: 10.1049/el:20010435. 
[39] Ederra I., Gonzalo R., Alderman B.E.J., Huggard P.G., de Hon B.P., van Beurden M.C., Murk A., Marchand L., DE MAagt P., Sub-millimeter-wave imaging array at $500 \mathrm{GHz}$ based on 3-D electromagnetic-bandgap material, IEEE Transactions on Microwave Theory and Techniques 56(11), 2008, pp. 2556-2565, DOI: 10.1109/TMTT.2008.2005926.

[40] Kesler M.P., Maloney J.G., Shirley B.L., Smith G.S., Antenna design with the use of photonic band -gap materials as all-dielectric planar reflectors, Microwave and Optical Technology Letters 11(4), 1996, pp. 169-174, DOI: 10.1002/(SICI)1098-2760(199603)11:4\%3C169::AID-MOP1\%3E3.0.CO;2-I.

[41] Smith G.S., Kesler M.P., Maloney J.G., Dipole antennas used with all-dielectric, woodpile photonic -bandgap reflectors: gain, field patterns, and input impedance, Microwave and Optical Technology Letters 21(3), 1999, pp. 191-196, DOI: 10.1002/(SICI)1098-2760(19990505)21:3\%3C191::AIDMOP10\%3E3.0.CO;2-L.

[42] Zhao Z., Deng Q., Xu H., Du C., Luo X., A sectoral horn antenna based on the electromagnetic band-gap structures, Microwave and Optical Technology Letters 50(4), 2008, pp. 965-969, DOI: $10.1002 /$ mop.23254.

[43] Weily A.R., Esselle K.P., Sanders B.C., Photonic crystal horn and array antennas, Physical Review E 68(1), 2003, article 016609, DOI: 10.1103/PhysRevE.68.016609.

[44] Moore R.L., Kesler M.P., Maloney J.G., Shirley B.L., Electromagnetic antenna and transmission line utilizing photonic bandgap material, US Patent 5689275, 1997.

[45] Weily A.R., Esselle K.P., SAnders B.C., Bird T.S., High-gain 1D EBG resonator antenna, Microwave and Optical Technology Letters 47(2), 2005, pp. 107-114, DOI: 10.1002/mop.21095.

[46] Weily A.R., Esselle K.P., SANDERS B.C., Layer-by-layer photonic crystal horn antenna, Physical Review E 70(3), 2004, article 037602, DOI: 10.1103/PhysRevE.70.037602.

[47] Weily A.R., Esselle K.P., Bird T.S., SAnders B.C., Linear array of woodpile EBG sectoral horn antennas, IEEE Transactions on Antennas and Propagation 54(8), 2006, pp. 2263-2274, DOI: 10.1109 / TAP.2006.879181.

[48] Liang M., Wu Z., NG W.R., Gehm M., Xin H., Electromagnetic crystal (EMXT) based terahertz horn antenna, [In] 2013 7th European Conference on Antennas and Propagation (EuCAP), Gothenburg, 2013, pp. 739-740.

[49] Colak E., Serebryannikov A.E., Ozgur Cakmak A., Ozbay E., Experimental study of broadband unidirectional splitting in photonic crystal gratings with broken structural symmetry, Applied Physics Letters 102(15), 2013, article 151105, DOI: 10.1063/1.4800147.

[50] Khromova I., Ederra I., Teniente J., Gonzalo R., Esselle K.P., Evanescently fed electromagnetic band-gap horn antennas and arrays, IEEE Transactions on Antennas and Propagation 60(6), 2012, pp. 2635-2644, DOI: 10.1109/TAP.2012.2194633.

[51] Foghani S., KaAtuzian H., Danaie M., Simulation and design of a wideband T-shaped photonic crystal splitter, Optica Applicata 40(4) 2010, pp. 863-872.

[52] KaAtuzian H., Danaie M., Foghani S., Design of a high efficiency wide-band 60 degree Y-branch for TE polarization, [In] 2009 14th OptoElectronics and Communications Conference, Vienna, 2009, pp. 1-2, DOI: $10.1109 /$ OECC.2009.5215096.

[53] Danaie M., Attari A.R., Mirsalehi M.M., Naseh S., Design of a high efficiency wide-band $60^{\circ}$ bend for TE polarization, Photonics and Nanostructures - Fundamentals and Applications 6(3-4), 2008, pp. 188-193, DOI: 10.1016/j.photonics.2008.08.003. 